\title{
Utilidad de la anestesia local del plexo periprostático en el control del dolor en las biopsias prostáticas guiadas con ecografia
}

\author{
J. Cervera Deval*, M. González Añón*, F. Martín Díez**, J. Jornet Fayos*, F.J. Morales Olaya*, \\ S. Selfa Moreno**
}

*Fundación Instituto Valenciano de Oncología. **Hospital Lluis Alcanyins. Xativa. Valencia.

Actas Urol Esp 2005; 29 (10): 943-947

\begin{abstract}
RESUMEN
UTILIDAD DE LA ANESTESIA LOCAL DEL PLEXO PERIPROSTÁTICO EN EL CONTROL DEL DOLOR EN LAS BIOPSIAS PROSTÁTICAS GUIADAS CON ECOGRAFÍA

Objetivo: La anestesia del plexo periprostático (APP) no es práctica habitual durante la biopsia prostática (BP). Muchos pacientes deben someterse a una segunda, o más, BP por sospecha de carcinoma. El dolor influye en la repetición de la misma pues determina que muchos pacientes rechacen el procedimiento o que éste deba realizarse en quirófano bajo anestesia. Nuestro objetivo fue valorar la utilidad de la APP en la supresión del dolor producido durante la realización de la BP así como la aceptación del procedimiento realizado en estas condiciones.

Material y métodos: De octubre del 2002 a junio del 2003 planteamos un trabajo prospectivo con casos y controles en el que incluimos aleatoriamente 275 pacientes de dos centros para BP. En el primer centro se incluyeron 101 varones a los que no se les realizó APP, mientras que en el segundo fueron 174 los pacientes sometidos a BP tras APP. Usamos hasta $10 \mathrm{ml}$ de lidocaína al $2 \%$ diluida al 50\% administrada mediante una aguja fina del calibre 22 . Al final del procedimiento se invitó al paciente a rellenar un cuestionario de satisfacción y evaluación del dolor.

Resultados: En el grupo de pacientes del segundo centro se obtuvieron valores medios significativamente inferiores $(\mathrm{p}<0,005$, IC 95\%) en la valoración cuantitativa del dolor, que en el grupo del primer centro $(1,24 \pm 0,4$ vs. $2,5 \pm 1,1)$. Respecto a la posibilidad, si fuese necesario, de repetir la BP, a ninguno de los pacientes del segundo centro le importaría, mientras que el $10 \%$ del primer centro no la repetirían o la aceptarían sólo bajo anestesia.
\end{abstract}

No se produjeron complicaciones atribuibles a la APP.

Conclusión: La APP es una técnica segura que reduce significativamente el dolor durante la BP, mejorando la aceptación de la misma.

Palabra clave: Dolor. Anestesia local. Ultrasonidos. Transrectal. Próstata. Biopsia.

\section{ABSTRACT}

UTILITY OF LOCAL ANESTHESIA OF THE PERIPROSTATIC PLEXUS IN THE CONTROL OF THE PAIN IN THE ULTRASOUND-GUIDED PROSTATE BIOPSY.

Objetive: Periprostatic plexus anesthesia (PPA) is not current practice during prostate ultrasound-guided biopsy (PB). Many patients must undergo a second or more PB if a prostate carcinoma is suspected. Due to pain, many patients reject the procedure, or it has to be performed with general anesthesia. Our objective was to evaluate the utility of PPA to eliminate the pain caused by PB, as well as the acceptance of the procedure under these conditions.

Patients and Methods: Between october 2002 and june 2003 we designed a randomized prospective study with 275 patients that were seen in 2 different hospitals and required PB. In the 1st group we included 101 males who underwent PB without PA. In the other group, 174 male patients submitted PB after PPA were included. 10 cc lidocaine, 2\% diluted $50 \%$ was injected with a $22 \mathrm{~g}$ needle. At the end of the procedure, patients were asked to fill in a questionnaire about their satisfaction and degree of pain felt.

Results: In the second group of patients we obtained significantly inferior values $(\mathrm{p}<0.005$, IC $95 \%)$ in the quantitative evaluation of pain compared with the first group (1.24 \pm 0.4 vs. $2.5 \pm 1$. 1). Ask per the possibility of repeating PB if it were necessary, none of the patients in the 2 nd group would object, whereas $10 \%$ in the 1st group would not have the biopsy repeated or would only accept it if it was done with general anesthesia. No complications due to PPA were found.

Conclusion: PPA is a safe procedure that significantly reduces pain during PB, improving its acquiescence among patients.

Keywords: Pain. Local anesthesia. Ultrasound. Transrectal. Prostate biopsy. 
$\mathrm{L}$ a Asociación Internacional para el Estudio del Dolor (IASP) define el dolor como una experiencia sensorial y emocional desagradable con daño tisular actual o potencial o descrito en términos de dicho daño ${ }^{1}$. El dolor es subjetivo, y es lo que el paciente dice que es, ya que en muchas ocasiones se refiere dolor, incluso en ausencia de daño tisular.

En 1989 Torp-Pedersen et al. ${ }^{2}$ introdujeron la Biopsia Prostática Transrectal guiada con ultrasonidos (TRUS) como método de diagnóstico en la sospecha de cáncer prostático. Es práctica habitual no anestesiar el plexo periprostático (PP) cuando se realiza una $\mathrm{BP}^{3}$ ya que se ha considerado que las biopsias transrectales no eran dolorosas ${ }^{4}$. Sin embargo, se ha publicado que hasta el 90\% de los pacientes de algunas series refieren molestias o dolor tras la biopsia transrectal ${ }^{3,4}$. Por otra parte, no es infrecuente tener que someter a un mismo paciente a un segundo o más procedimiento de BP por sospecha de carcinoma. Por tanto, múltiples estudios han demostrado la conveniencia de controlar el dolor cuando se realiza una biopsia prostática transrectal $(\mathrm{BP})^{5,6}$. El dolor influye directamente en la confortabilidad del procedimiento y, por tanto, facilita o entorpece la repetición del mismo, siendo muchos los casos en los que el paciente se niega a ello o en los que debe ser repetido en quirófano bajo anestesia.

Nuestro objetivo ha sido triple. En primer lugar valorar el dolor producido cuando realizamos una BP por sextantes en pacientes con sospecha de carcinoma de próstata. En segundo lugar valorar la eficacia de la anestesia del PP en la supresión del dolor $\mathrm{y}$, finalmente, el grado de aceptación del procedimiento por parte de los pacientes cuando se realiza bajo anestesia del PP.

\section{MATERIAL Y MÉTODOS}

Durante un periodo de nueve meses (octubre 2002 a junio 2003) hemos realizado un trabajo prospectivo con casos y controles en el que incluimos de forma prospectiva y aleatoria a los pacientes de dos centros, candidatos a la realización de BP, que tras el consentimiento informado aceptaron su inclusión para la realización del procedimiento. En el Primer Centro se incluyeron 101 varones a los que no se anestesiaba local- mente el PP, mientras que en el Segundo Centro la muestra estuvo constituida por 174 varones a los que se les practicó la BP tras anestesiar el PP. Esto se realizó mediante la inyección, con aguja de calibre $22 \mathrm{G}$, de hasta $10 \mathrm{ml}$ de lidocaína al $2 \%$ diluida al $50 \%$ con suero fisiológico, que se aplicó mediante guía ecográfica entre la pared rectal y la próstata, distribuyéndolo a nivel del ápex y márgenes posterolaterales siguiendo a otros autores $^{4,7,8}$. La BP se obtuvo pasados 3 minutos, después de la aplicación anestésica, mediante una aguja del calibre $18 \mathrm{G}$, de disparo automático y de $20 \mathrm{~cm}$ de longitud.

Todos los pacientes recibieron protección antibiótica previa y posterior al procedimiento, consistente en Ciprofloxacino oral $500 \mathrm{mg}$ cada 12 horas, durante 5 días, comenzando el día antes. La técnica de realización de la BP fue similar en ambos Centros, consistiendo en la obtención de muestras por sextantes y la adición de más muestras cuando había alguna zona sospechosa.

En ambas series las indicaciones de BP se basaron en la elevación de PSA $>4 \mathrm{ng} / \mathrm{ml}$ o por un tacto rectal sospechoso en la exploración urológica. Se excluyeron a 12 pacientes por rehusar el procedimiento o por padecer en el momento del estudio enfermedad inflamatoria rectal.

En ningún momento el paciente fue conocedor del uso del anestésico local.

Al final del procedimiento y una vez transcurridos 15-30 minutos, antes de despedir al paciente, y mediante el soporte del personal de enfermería, se le invitaba al mismo a rellenar un cuestionario de satisfacción y evaluación del dolor, en los que el dolor estaba gradado en una escala de 0 a 5, acorde con la intensidad de dolor, de modo que 0 era ningún dolor y 5 dolor insoportable. En dicho cuestionario, y por razones metodológicas, la pregunta de interés quedó enmascarada (Ver Encuesta Biopsia Prostática) (Fig. 1).

Para el manejo de datos se recodificaron dos grupos. En el primero (grupo I) clasificamos aquellos con dolor moderado, severo o insoportable, mientras que los que refirieron ningún o poco dolor, los clasificamos en el segundo grupo (grupo II). Los datos recogidos en el cuestionario dado a los pacientes los analizamos con pruebas no paramétricas como la U de Mann-Whitney, Chi 


\begin{tabular}{|c|}
\hline ENCUESTA DE BIOPSIA PROSTÁTICA \\
\hline NOMBRE: \\
\hline PROFESIÓN: \\
\hline ESTUDIOS: \\
\hline $\begin{array}{l}\square \text { Elementales } \\
\text { Grado Medio } \\
\square \text { Grado Superior }\end{array}$ \\
\hline $\begin{array}{l}\text { ¿CÓMO LE PARECIÓ LA ILUMINACIÓN DE LA } \\
\text { SALA? }\end{array}$ \\
\hline $\begin{array}{l}\square \text { ESCASA } \\
\square \text { ADECUADA } \\
\square \text { EXCESIVAA }\end{array}$ \\
\hline ¿HA SENTIDO DOLOR? \\
\hline $\begin{array}{l}\square \text { NINGÚN DOLOR } \\
\square \text { POCO DOLOR } \\
\square \text { BASTANTE DOLORÅ } \\
\square \text { MUCHO DOLOR } \\
\square \text { INSOPORTABLE }\end{array}$ \\
\hline LA POSICIÓN DE LA CAMILLA FUE: \\
\hline $\begin{array}{l}\square \text { Confortable } \\
\square \text { Incomoda } \\
\square \text { Muy incomoda }\end{array}$ \\
\hline SI SE TUVIERA QUE REPETIR LA PRUEBA \\
\hline $\begin{array}{l}\text { No me importaría repetirla } \\
\text { Si fuese necesaria me gustaría con anestesia } \\
\text { general } \\
\text { No la repetiría bajo ningún concepto }\end{array}$ \\
\hline SUGERENCIA: \\
\hline
\end{tabular}

\section{FIGURA 1}

cuadrado y el test de Fischer, con $95 \%$ de intervalo de confianza $(p<0,05)$, cuando eran apropiados. Además analizamos la influencia de la edad, recodificándola en dos categorías según sean mayores o menores de 65 años, para la presencia o ausencia de dolor, mediante un análisis de regresión logística.

\section{RESULTADOS}

Del total de 275 varones incluidos, 101 correspondieron al Primer Centro y 174 al Segundo Centro. Las distribuciones de edad, volumen prostático, PSA, número de biopsias realizadas y resultados anatomopatológicos obtenidos, están resumidas en la Tabla 1.
Tabla 1

\begin{tabular}{lcc}
\hline Variable & $\begin{array}{c}\text { Primer } \\
\text { centro }\end{array}$ & $\begin{array}{c}\text { Segundo } \\
\text { centro }\end{array}$ \\
\hline Anestesia & $\mathrm{NO}$ & $\mathrm{SI}$ \\
No Pacientes & 101 & 174 \\
Edad (media \pm DS) & $68 \pm 5,8$ & $65 \pm 8,2$ \\
Volumen Prostático & $\mathrm{n} . \mathrm{e}$ & $47 \pm 27,3$ \\
(media \pm DS) & $6,76 \pm 1,6$ & $14.8 \pm 46,43$ \\
PSA (media \pm DS) & $6,2 \pm 0,9$ & $6 \pm 0,8$ \\
№ Biopsias realizadas & $(M x=12, M n=5)$ & $(M x=12, M n=5)$ \\
(media \pm DS) & 28 & 52 \\
AP de Malignidad & 73 & 122 \\
AP de Benignidad & \multicolumn{2}{c}{} \\
\hline n.e.- Dato no especificado & \multicolumn{2}{c}{}
\end{tabular}

No se produjo ninguna complicación atribuible, al menos inmediata, a la aplicación del anestésico local.

En el grupo de pacientes del Segundo Centro, que llevaron anestesia local, se obtuvieron valores medios significativamente menores $(\mathrm{p}<0,005$, IC 95\%) en la valoración cuantitativa del dolor $(1,24 \pm 0,4)$, que en los casos incluidos en el Primer Centro $(2,5 \pm 1,1)$, a los que no se infiltró con anestésico local.

Sólo el 2,3\% (4/174) de los pacientes a los que se les aplicó el anestésico local refirieron dolor moderado, severo o insoportable, mientras que encontramos 31 pacientes con dolor de esta intensidad, de un total de $101(30,6 \%)$ a los que no se les aplicó el anestésico local $(\mathrm{p}<0,001)$.

Respecto a la posibilidad de repetir el procedimiento, si fuera necesario, a ninguno de los pacientes a los que se les había aplicado anestesia local, le importaría su repetición, no encontrando referencias a la necesidad de anestesia general o a una negativa radical a su repetición. Por el contrario, de los pacientes incluidos sin anestesia local, 10 (10\%) refirieron un dolor insoportable, de los cuales 8 señalaron necesitar anestesia general para su repetición y 2 no estarían dispuestos a su repetición.

\section{DISCUSIÓN}

Para valorar la respuesta al tratamiento del dolor, dadas las características subjetivas del mismo, se han diseñado y utilizado diferentes cuestionarios y escalas, cada una de ellas para una aplicación específica. Así, el McGill Pain Questionnaire ${ }^{9}$, un cuestionario para medir el dolor, se usa en numerosos estudios clínicos, siendo un método fiable para evaluar el dolor crónico. Otro método de cuantifi- 
car el dolor es mediante las escalas numéricas (Numerical Rating Scale-NRS), las cuales valoran en un rango de 0 a 10 la intensidad de dolor, de modo que el valor 0 expresaría la "Ausencia de Dolor" y el 10 lo "Peor posible". Similar es la Escala Visual Analógica -VAS, que consiste en una línea de $10 \mathrm{~cm}$ dividida en 10 segmentos de $1 \mathrm{~cm}$, comenzando por 0 (no dolor) y en el lado opuesto el 10 (lo peor posible). En ésta, el paciente marca en la línea un punto con el que él cuantificaría la intensidad de dolor. La VAS y NRS son de uso universal y se utilizan para valorar la reducción del dolor después de aplicar el tratamiento.

Nosotros hemos utilizado una escala descriptiva simple, similar a la utilizada por otros autores ${ }^{3,4}$, por su rapidez en la encuesta, así como facilidad de uso y comprensión por parte del paciente. Esta escala consiste en una sencilla clasificación verbal en categorias, normalmente en 4 ó 5 categorias (ausente, leve, moderado, intenso o insoportable). Por ello, asumiendo que el paso de una categoría a otra puede representar cosas diferentes, para el análisis estadístico hemos utilizado pues, pruebas no paramétricas para la obtención de conclusiones.

Los nervios del paquete neurovascular que inervan la próstata discurren por el margen posterolateral de la glándula, atravesando la cápsula en las posiciones 4 y 8 del reloj. Pero además, el control del dolor es más efectivo cuando el anestésico difunde amplia y libremente ${ }^{4}$. Durante mucho tiempo se ha considerado que la biopsia transrectal no era dolorosa ${ }^{4}$, por lo que es práctica habitual realizarla sin ninguna clase de anestesia o sedación $^{3,7,10}$. Sin embargo, en nuestra experiencia y en la de otros autores esto no es cierto, llegando a ser definida por algunos como una "experiencia traumática y dolorosa"3. Así, el grupo de Seymour H et al. describen que hasta el $30 \%$ de los pacientes refieren dolor de diversa severidad durante la $\mathrm{BP}^{4}$. En el mismo sentido, y categorizando el dolor, el $16 \%$ de casos de la de Irani et $\mathrm{al}^{6}$, en las que no se aplicó anestesia local, presentaron una valoración de VAS > 5. Crundwell et al. encuentran que el $24 \%$ de los pacientes de su serie califican el procedimiento como "moderado a extremadamente doloroso" ${ }^{10}$, resultados que coinciden con los nuestros en los que el 30,6\% de los pacientes no anestesiados adscribieron el dolor a las categorías de moderado a insoportable, cifras que, sin embargo, son muy superiores en otras series como la de Kaver $\mathrm{I}^{7}$ o la de Tobias Machado et al. ${ }^{3}$ alcanzando en esta última hasta el 90\% de los pacientes no anestesiados. Los resultados obtenidos por nosotros confirman una mejoría significativa en cuanto a la percepción del dolor durante la BP realizada con anestesia local, obteniendo resultados bajos en la escala de dolor, sensiblemente inferiores a los obtenidos en el grupo no anestesiado (1,24 vs. $2,5, p<0,005)$. Resultados similares se obtienen por otros grupos como el de Seymour $\mathrm{H}$ et al. (1,53 vs 1,95 , $p<0,001)^{4}$, el de Addla SK et al. $(3,0$ vs 4,3 , $p<0,001)^{8}$ o el de Von Knobloch $(1,85$ vs 3,29 , $p<0,0001)^{11}$. En nuestra serie sólo el 2,3\% (4/174) de los pacientes anestesiados adscribieron el dolor percibido a la categoría "moderado-insoportable", y ninguno de ellos refirió el grado máximo ("insoportable"), lo que también concuerda lo obtenido por otros $^{3,7}$ (Tabla 2).

\section{Tabla 2}

Distribución de la Aceptación según el grado de dolor

\begin{tabular}{|c|c|c|c|c|c|}
\hline Anestesia & Valoración & & $\begin{array}{c}\text { No Importaría } \\
\text { Repetirla } \\
\mathbf{N}\end{array}$ & $\begin{array}{c}\text { Necesitaría } \\
\text { Anestesia General } \\
\text { N }\end{array}$ & $\begin{array}{c}\text { No Repetiría } \\
\mathbf{N} \\
\end{array}$ \\
\hline \multirow[t]{5}{*}{ SI } & 1 & Ningún Dolor & 137 & & \\
\hline & 2 & Poco Dolor & 33 & & \\
\hline & 3 & Bastante Dolor & 4 & & \\
\hline & 4 & Mucho Dolor & & & \\
\hline & 5 & Dolor Insoportable & & & \\
\hline \multirow[t]{6}{*}{ NO } & Valoración & & & & \\
\hline & 1 & Ningún Dolor & 8 & & \\
\hline & 2 & Poco Dolor & 62 & & \\
\hline & 3 & Bastante Dolor & 14 & & \\
\hline & 4 & Mucho Dolor & 7 & & \\
\hline & 5 & Dolor Insoportable & 2 & 6 & 2 \\
\hline
\end{tabular}


Respecto al impacto del dolor en la aceptación del procedimiento, se ha descrito que el dolor desanima a aquellos pacientes con "PIN de alto grado", o sospecha clínica persistente de cáncer no identificado, a aceptar la repetición de biopsias $^{10}$. Así, se ha publicado que hasta el $19 \%$ de los varones rechazan la rebiopsia prostática por una experiencia inicial dolorosa ${ }^{6}$. Sin embargo, la aceptación de la biopsia prostática como procedimiento diagnóstico es muy importante ya que, como señalan algunas series, la rebiopsia prostática puede aumentar la detección del cáncer prostático hasta en un $30 \%$ de los $\operatorname{casos}^{10}$. Aunque algunas series no encuentran diferencias significativas respecto al grado de aceptación del procedimiento ${ }^{4}$, nuestros resultados muestran que a ninguno de los pacientes a los que se les aplicó anestesia local les importaría someterse a una nueva biopsia, frente al $10 \%$ del grupo no anestesiado que, o bien rehusaría siempre la rebiopsia, o sólo la aceptaría bajo los efectos de una anestesia general. Estos resultados concuerdan también con los obtenidos por otros autores. Así, Tobias Machado et al. obtienen un 100\% de aceptación de la rebiopsia en el grupo anestesiado vs. sólo un $45 \%$ en el grupo no anestesiado ${ }^{3}$.

Además, aunque existen discrepancias en la literatura a este respecto ${ }^{12}$, en nuestra experiencia y en la de otros, el dolor aumenta con el número de pases biópsicos realizados ${ }^{7,13}$ y esto ha sido reconocido como una limitación significativa a aumentar el número de pases con el fin de minimizar el "error de muestreo"4 . El bloqueo de los nervios del paquete neurovascular que inervan la próstata con Lidocaína obtiene una reducción subjetiva de la sensación de dolor, pudiendo de este modo realizar, según algunas series, hasta 10 ó 15 pases, sin inducir un incremento del dolor y con una buena aceptación por parte del paciente ${ }^{7,10}$. Nosotros, en nuestro estudio, hemos llegado a hacer hasta 12 pases por paciente sin incremento en la percepción del dolor en estos casos, lo cual adquiere una particular importancia en aquellos pacientes que son remitidos para rebiopsia y en los cuales existe una tendencia a incrementar el número de pases con el fin de minimizar el ya reseñado "error de muestreo". Finalmente, somos concordantes con las series en las que no encuentran diferencias significativas en cuanto a la edad ${ }^{7,14}$. En nuestro estudio la hemos dicotomizado en menor o mayor de 65 años, y respecto a la presencia o ausencia de dolor, sin o con anestesia, no encontrando diferencias significativas.

En resumen, al igual que otros autores, creemos que el uso de anestesia local periprostática durante la realización de una $\mathrm{BP}$, además de ser un método fácil y seguro, disminuye o suprime el dolor producido por el procedimiento, permite aumentar el número de pases e incrementa la aceptación del mismo, por lo que debería ser incluida de forma sistemática como paso previo inmediato a la realización de la BP.

\section{REFERENCIAS}

1. International Association for the Study of Pain (IASP): http://www.iasppain.org/terms-p.html, Acceso 29 de agosto de 2003.

2. Torp-Pedersen S, Lee F, Littrup PJ, Siders DB, Kumasaka GH, Solomon MH, McLeary RD. Trans-rectal biopsy of the prostate guided with transrectal US: longitudinal and multiplanar scanning. Radiology 1989;170:23

3. Machado MT, Aragäo AJ, Rodríguez AO, Wroclawski ER. Periprostatic local anestesia in Transrectal ultrasound-guided prostate biopsy: is it posible to improbé pain tolerance?. Int Braz J Urol 2002;28:323-329.

4. Seymour H, Perry MJA, Lee-Elliot C, Dundas D, Patel U. Pain after transrectal ultrasonography-guided prostate biopsy: the advantages of periprostatic local anaesthesia. BJU International 2001;88:540-544.

5. Collins GN, Lloyd SN, Hehir M, McKalvie GB. Multiple transrectal ultrasound-guided prostatic biopsies:true morbidity and patient acceptance. $\mathrm{Br}$ J Urol 1993;71:460-463.

6. Irani J, Fournier F, Bon D, Gremmo E, Dore B, Aubert J. Patient tolerance of transrectal ultrasound-guided biopsy of the prostate. Br J Urol 1997;79: 608-610.

7. Kaver I, Mabjeesh NJ, and Matzkin H. Randomized prospective study of periprostatic local anesthesia during transrectal ultrasound-guided prostate biopsy. Urology 2002;59:405-408.

8. Addla SK, Adeyoju AAB, Wemyss-Holden GD, Neilson D. Local anaesthetic for transrectal ultrsound-guided prostate biopsy: A prospective, randomized, double blind, placebo-controlled study. European Urology 2003; 441443.

9. Melzack R. Pain - an overview. Acta Anaesthesiol Scand 1999;43:880-884.

10. Crundwell MC, Cooke PW, Wallace DMA. Patient's tolerance of transrectal ultrasound-guided prostatic biopsy: an audit of 104 cases. BJU International 1999;83:792-795.

11. Von Knobloch R, Weber J, Varga Z, Feiber H. Bilateral fine-needle administered local anaesthetic nerve block for pain control during TRUS-Guided multi-core prostate biopsy: A prospective randomised Trial. Eur Urol 2002;41:508-514.

12. Naughton CK, Ornstein DK, SmithDS, Catalona WJ. Pain and morbidity of transrectal ultraound guided prostate biopsy: a prospective randomized trial of 6 versus 12 cores. J Urol 2000;163:168-171.

13. Soloway MS, Obek C. Periprostatic local anaesthesia before ultrasound guided prostate biopsy. J Urol 2000;163:172-173.

14. Berger AP, Frauscher F, Halpern EJ, Spranger R, Steiner H, Bartsch G, Horninger W. Periprostatic administration of local anesthesia during transrectal ultrasound-guided biopsy of the prostate: a randomized, double-blind placebo-controlled study. Urology 2003;61:585-588.

Dr. J. Cervera Deval

Servicio de Radiología. Inst. Valenciano de Oncología

Profesor Beltrán Báguena, 19 - 46006 Valencia

E-mail: jcervera@telefonica.net

(Trabajo recibido el 5 de marzo de 2005) 